\title{
La regulación de la
} publicidad programática online: un análisis desde el prisma del derecho comparado*

\author{
Alex SosA ${ }^{1}$
}

\section{RESUMEN}

En el actual mercado de publicidad online, los agentes económicos buscan desviar la demanda de los consumidores hacia sus ofertas a través del uso de publicidad programática. Este tipo de publicidad que hace uso del big data e inteligencia artificial permite que las empresas puedan enviar publicidad a los consumidores realmente interesados en sus productos, lo cual incrementa la posibilidad de un "matcb" entre ellos. Si bien este tipo de publicidad puede generar como efecto el incremento de la competencia y oferta en beneficio de los consumidores, también puede generar riesgos importantes para el correcto funcionamiento del mercado si se utilizan como práctica desleal (incluyendo infracción de derechos de marca) y perjuicios para los usuarios si no conocen el uso que se hace de los datos que comparten de forma voluntaria o involuntaria. En el presente artículo analizaremos cómo funciona esta estrategia publicitaria, los beneficios y riesgos que implica, así como también la forma en la que viene enfrentando dichos riesgos en Estados Unidos y países de la Unión Europea. Con este artículo, el autor también tiene como finalidad motivar una discusión mucho más profunda a nivel sudamericano.

Palabras clave: protección al consumidor, competencia desleal, publicidad, publicidad programática o conductual, big data, palabras clave, signos distintivos, Google Ads, Facebook Ads, derecho económico internacional, mercado global, datos personales, privacidad.

1 Profesor TC de la Universidad Científica del Sur. Consultor Legal, Lima, Perú. Magíster en Derecho de la Propiedad Intelectual y de la Competencia por la Pontificia Universidad Católica del Perú. Abogado Summa Cum Laude por la Universidad de Lima. Correo electrónico: asosah@pucp.pe. ORCID: https://orcid.org/0000-0003-2122-3329

* DOI: https://doi.org/10.18601/01236458.n56.07 


\section{ABSTRACT}

In the current market of online advertising, economic agents look to redirect the consumers demand to their products through the use of programmatic advertising. This type of advertising that uses Big Data and artificial intelligence, allows the companies to send advertisements to the consumers that are really interested in their products, which increases the possibility of a "match" between them. While this type of advertising can generate the competition and supply increase to the benefit of the consumers as an effect, it can also generate important risks for the correct operation of the market if they are used as unfair practice (including trademark rights infringement) and damages for the users if they ignore what is done with the data they willingly or unwillingly share. In this article we will analyze how this marketing strategy work, the benefits and risks that it represents, as well as the way such risks are being faced in the United States and European Union countries. The author aims to motivate a much deeper discussion at the South American level.

Keywords: Consumer Protection, Unfair competition, Advertising, behavioural advertising, big data, key words, Trademarks, Google Ads, Facebook Ads, International Economic Law, global market, personal data, privacy.

\section{INTRODUCCIÓN}

Existen varios medios online a través de los cuales se puede ejecutar y difundir la publicidad programática, por lo que un desarrollo completo podría exceder el objetivo de este trabajo. Es por ello que nos concentraremos en la publicidad programática que nos llega a través de Google, redes sociales (abordando dos de las más grandes como Facebook e Instagram, que tienen el mismo origen empresarial) y la comportamental o conductual, la cual recibimos producto de nuestra actividad en la Web. Así, buscaremos desarrollar la evolución de la publicidad comercial online y definiremos los tipos de publicidad programática que existen, con el objeto de exponer los beneficios que genera esta estrategia para los mercados y consumidores, sin dejar de lado los riesgos que podría generar en los derechos de los consumidores.

Para efectos del presente trabajo, tomaremos como referencia de análisis los intentos de regulación de la publicidad programática en Estados Unidos y países de Europa, en la medida que en sus jurisdicciones es donde podemos encontrar la mayor evidencia de estudio de su regulación. Es importante precisar que el estudio de la publicidad programática y sus efectos en el mercado y consumidores aún es incipiente en países distintos a los antes señalados, por lo que en el presente trabajo no tomaremos en consideración estudios o casos que se hayan llevado a cabo en ellos.

Asimismo, es importante precisar que si bien el presente artículo tiene como uno de sus objetivos cuestionar si es necesaria la regulación de la publicidad programática, el autor tiene como finalidad motivar una discusión mucho más profunda a 
nivel sudamericano. En ese sentido, el análisis jurídico, si bien desarrolla temas de competencia desleal, marcas, privacidad y datos personales, se concentra en mayor medida en los efectos de una eventual censura o intervención excesiva por parte de la regulación estatal.

\section{EVOLUCIÓN DE LA PUBLICIDAD COMERCIAL: LA PUBLICIDAD PROGRAMÁTICA}

El uso adecuado del big data por parte de las empresas ha generado en muchos casos que puedan lograr una ventaja significativa en el mercado y esto a la vez, se ha visto reflejado en un incremento de la competencia en los mercados y más información en beneficio del consumidor, lo cual ha venido acompañado también de riesgos a su privacidad. Al fenómeno del big data lo caracteriza la inmensidad de datos estructurados y no estructurados que lo conforman, al ser toda la información que generamos como humanidad conectada a internet través de cualquier tipo de nodo (dispositivo con conexión a internet), la cual es imposible de condensar en su totalidad, debido a su gran volumen, variabilidad y velocidad de crecimiento, sin embargo, dicha información, bien o mal utilizada, podría generar ventajas importantes por la predictibilidad de comportamientos y sucesos que pueden generarse (Gil, 2016).

En efecto, en los últimos años, gobiernos, partidos políticos, empresas, entre otros, vienen desarrollando proyectos que hacen uso del big data con el claro objeto de desarrollar eficiencias en virtud de la información con la cual pueden contar. Así, un proyecto de big data, que se puede desarrollar a través de uso de algoritmos, entre otras fuentes, le permite predecir comportamientos y demanda de sus potenciales clientes y competidores, sobre la base de la información que se encuentra en la nube, la que le comparten sus clientes (o socios comerciales) y las que generamos los usuarios de Internet con nuestro comportamiento online. Dicha información le permite conocer a las empresas qué es lo que realmente demandan los consumidores interesados en el producto o servicio que oferta en el mercado y así poder adaptar su oferta a los gustos de su público objetivo (Townley et al., 2017).

Un gran ejemplo que podemos mencionar es Netflix, que a través de su algoritmo puede tener información acerca de lo que realmente demandan sus suscriptores segmentando perfiles. Ello le permite poder producir contenido a la medida de lo que desean ver sus diversos usuarios, lo cual le ha servido para posicionarse en el mercado mundial de producción de series y películas — muchas de ellas premiadas - (ClickZ, 2019). Por su parte, Inditex tiene el control de todas las tiendas de Zara, gracias a su proyecto de big data, el cual le permite tener información real sobre las tallas más demandadas por zonas geográficas, tener un inventario y stock perfecto, así como establecer las modas de acuerdo con lo que los consumidores más demandan. Es decir, los diseñadores ya no imponen las modas, las imponemos nosotros (Uberoi, 2017).

Cada vez más tiendas y supermercados utilizan los datos que tienen a su disposición para poder ofrecer directamente a sus clientes promociones sobre los productos que más adquieren, en virtud del registro de nuestras compras online (carritos de compra) 
o las ofertas que hemos revisado por sus redes sociales, lo cual le permite poder enviar publicidad y promociones específicas sobre los productos que realmente les interesan a sus clientes. Ciertamente, el uso de grandes cantidades de datos es mucho más que una herramienta útil para la toma de decisiones, pues el contar con esa metodología y esas técnicas de investigación del análisis de datos proporciona una visión única y puede generar una importante ventaja competitiva, dependiendo del contexto en el que se lleve a cabo, como en la política, la ciencia o los negocios (Téllez, 2020).

Este desarrollo en el uso del big data genera beneficios para los consumidores en la medida que se incrementa la oferta y competencia entre agentes económicos, pero sobre todo, genera beneficios importantes para las empresas, toda vez que con el uso de la información que ahora tiene a su disposición (o puede obtener del comportamiento online), pueden dirigir mejor su publicidad comercial a audiencias específicas que se encuentren realmente interesadas en sus productos, incrementando de esta forma la posibilidad de generar el match entre proveedores y consumidores. Todo ello, en virtud de los datos que pueden compilar de forma directa o indirecta y del uso del algoritmo correcto que le permita utilizar la información que dispone a su favor para llegar a los consumidores que demandan los productos o servicios que oferta en el mercado. Y es que el uso de esta información permite a las empresas segmentar y definir grupos demográficos con mayor precisión que las estrategias tradicionales. Con la información que les proporcionamos y la que puede obtener de nuestro comportamiento online, los operadores económicos pueden conocer nuestros intereses especiales y actividades que desplegamos durante el día o noche con el objeto de enviarnos publicidad. Así, estas empresas pueden segmentar por edad, sexo y clase social, lo cual de por sí, también implica un riesgo (Fletcher, 2010).

Un agente económico que utiliza eficientemente la información que puede obtener del big data puede optimizar sus procesos de venta o concurrencia en el mercado a través de un mejor nivel de predictibilidad del comportamiento de los consumidores, lo cual le puede generar una ventaja competitiva importante en el mercado. Sobre esto último, Sánchez (2019) señala que

la nueva dinámica competitiva ha llevado a distintas compañías a realizar un análisis más fino acerca de los gustos y/o preferencias de sus usuarios o clientes, con el objetivo de proponerles soluciones más ajustadas a sus necesidades o exigencias. El Big Data permite a las compañías, más que acceder a ingentes cantidades de datos, encontrar esas "pepitas de oro", esos datos de calidad que nos permitan mejorar su posición competitiva con la generación de productos cada vez más personalizados. Esta dinámica de personalización ha permitido que los usuarios tengan una parte activa en la producción de bienes y servicios, ya que ahora las compañías pueden integrarlos, a través de sus datos, como una parte importante de la cadena de desarrollo e innovación de sus productos o servicios. (pp. 146-147) 
Así, en un contexto de competencia encarnizada en la que cada día existen más incentivos para que los operadores económicos inviertan en investigación y nuevas tecnologías, tenemos que la forma de hacer publicidad comercial ha evolucionado completamente, al ser la principal herramienta de competencia con la que cuentan las empresas (Fletcher, 2010).

En efecto, para que su publicidad sea más efectiva, los anunciantes han reducido la compra de espacios publicitarios en los programas más demandados de los medios de comunicación tradicionales como la TV y radio, para ahora comprar audiencias e invertir en la publicidad digital (Carrillo-Durán y Rodríguez, 2018). Y es que a través de la publicidad por Internet puede obtener información acerca de las preferencias y gustos de los consumidores de forma directa (entregada voluntaria o involuntariamente) o a través de su comportamiento en la Web (muchas veces involuntaria y desinformada). Asimismo, puede dirigir publicidad comercial a determinados perfiles los cuales podrá segmentar con mayor facilidad que con el uso de medios de comunicación tradicionales, así como también puede tener una comunicación interactiva, incluso en tiempo real, con el consumidor, el cual ya no tiene una posición pasiva frente a la publicidad (López, 2013).

En el mundo del marketing, a esta evolución de la publicidad se le ha denominado publicidad programática, la cual, en líneas generales, podríamos definir como la publicidad que hace uso del big data para difundirse directamente a los consumidores que demandan el producto que oferta en el mercado el anunciante. A través de este tipo de publicidad, los anuncios se pueden orientar en función de los datos demográficos, contenido web, los intereses de los consumidores o su comportamiento de navegación online o su historial de compra de productos, entre otros factores (Lerner, 2014).

La publicidad programática, en principio, genera eficiencias para ambas partes, proveedores y consumidores, toda vez que permite incrementar la posibilidad de la existencia de un match entre ellos con menos gasto en publicidad para el proveedor y con más información para los consumidores. En efecto, para el anunciante, hace que su inversión en publicidad cada vez sea más efectiva y para el consumidor, le genera más información de acuerdo con sus reales intereses. Para probar ello, basta solo con comparar la cantidad y tipo de publicidad a la que estamos expuestos con la publicidad tradicional en TV y la publicidad por plataformas digitales. En los medios de comunicación tradicionales somos bombardeados con mucha publicidad de cosas que, en su mayoría, no necesitamos ni estamos interesados en adquirir, pues finalmente, si bien se trata de utilizar estrategias de segmentación de perfiles por contenido, lo cierto es que la gran mayoría de anunciantes busca comprar un espacio publicitario en los programas más demandados o de más rating. El que buena parte de la publicidad no llegue a las personas realmente interesadas en la oferta implica un costo hundido para el anunciante y una pérdida de tiempo para el consumidor. En cambio, con la publicidad programática, por ejemplo, Google utiliza los datos que le hemos entregado voluntariamente utilizando Chrome, el buscador de Google, Gmail, Youtube, 
etc. para enviarnos publicidad acorde a nuestros intereses mostrados en las referidas plataformas (Picker, 2009).

Ahora bien, es importante precisar que no todos los agentes económicos coinciden en considerar que esta estrategia publicitaria es más efectiva que la publicidad tradicional, los estudios de Chen y Stallaert (2010) evidencian que la publicidad programática si bien puede generar beneficios para agentes económicos de baja cuota de mercado o recién entrantes (ya que les puede hacer ganar rápidamente clientes), no se trata de una estrategia rentable para los agentes ya posicionados o dominantes de un determinado mercado relevante, quienes llegan a una buena cantidad de receptores a través de su inversión únicamente en publicidad tradicional. Así, los referidos autores señalan, además, que es costoso virar de la publicidad tradicional a la comportamental, por lo que no todos los agentes estarían dispuestos a realizar tal inversión, ya que su implementación implica el uso de más actores en la elaboración de la publicidad, inversión en algoritmos y cookies (pp. 35-37). Sin embargo, de acuerdo con los datos que contamos de la empresa Cyberclik (2020), para el 2018, un 82\% de las compañías que optaron por introducir la inteligencia artificial a través de publicidad programática notaron un aumento positivo en su retorno sobre la inversión (p. 75).

No obstante, lo cierto es que con el uso de los medios de comunicación tradicionales para difundir publicidad, los anunciantes destinan buena parte de sus recursos a difundir publicidad jugando un poco al azar (a pesar de hacer estudios de mercado de segmentación de perfiles), pues al dirigir su publicidad comprando espacios publicitarios de los lugares más demandados o concurridos por los consumidores, consideran que su inversión dará resultados. El problema de esta estrategia es que es muy difícil determinar qué parte de esos recursos invertidos termina siendo solo un gasto y qué parte efectivamente genera un retorno económico. La publicidad programática prácticamente ha eliminado ese problema, ya que gracias a los datos de nuestros intereses y/o comportamiento en la web, los anunciantes pueden dirigir su publicidad a quienes realmente demanden su oferta. Las empresas obtienen dichos datos de las siguientes formas: (i) obtiene la información directamente y de forma voluntaria por parte de los propios consumidores (información entregada a aplicaciones o plataformas digitales), (ii) utiliza data de consumidores ubicadas en plataformas privadas (por ejemplo, redes sociales, las cuales también son entregadas voluntariamente); y, (iii) fuentes disponibles públicamente, que van desde registros públicos del gobierno (como títulos de propiedad real) hasta recopilación automática de datos publicados en sitios web (por ejemplo, correos electrónicos de abogados de un despacho, como su trayectoria profesional) o navegaciones en la web (a través de uso de tracking cookies). Esto último, incluye la estrategia de colección de datos denominada scraping, que consiste en el uso de herramientas automatizadas (llamadas robots o arañas) que escarban en páginas web para obtener datos (Tushnet y Goldman, 2014), como también técnicas de marketing de base de datos o minería de datos, entre otras técnicas electrónicas que tienen por objeto segmentar los perfiles de los consumidores, como el database 
marketing, data mining, computer profiling y el front end verification, las cuales López Jiménez (2013) las define del siguiente modo:

Respecto al database marketing (o marketing de base de datos), cabe decir que está especialmente vinculado con la relación que se suscita entre el anunciante y el receptor de la publicidad. Busca desarrollar una base de datos que incluya tanto a los consumidores actuales como a los potenciales [...].

El data mining (o minería de datos), que prepara, sondea y explora los datos, para sacar información oculta presente en los mismos, persigue hacerse con determinad información personal de los potenciales consumidores y/o usuarios para promocionar ciertos productos y/o servicios. Así, por ejemplo, mediante esta técnica se analiza el comportamiento de los visitantes en un sitio web, y, sobre la base de los productos adquiridos de los consumidores con anterioridad, se les oferta otros en los que, en atención a su perfil, se prevé que pudieran estar interesados.

El computer profiling permite efectuar una combinación de datos elementales que aisladamente considerados no significarían nada, por lo que pasarían desapercibidos para los anunciantes, pero que relacionados dan la oportunidad de conocer perfiles comerciales de los clientes.

Finalmente, la práctica denominada front end verification es utilizada para contrastar la exactitud de la información personal cotejándola con informaciones similares almacenadas en bases de datos informáticas generalmente de terceros. (pp. 224-227)

La gestión de estos datos es completamente relevante para el desarrollo de la publicidad programática. Cabe señalar que no estamos hablando del mero uso de datos para la difusión de publicidad segmentada a perfiles. Estamos frente a una estrategia publicitaria que hace uso de la tecnología para procesar y gestionar datos, lo cual, desde el punto de vista comercial, puede generar eficiencias importantes para el mercado de la publicidad como lo señalan Carrillo-Durán y Rodríguez (2018):

La gestión de datos en publicidad programática aporta grandes ventajas a todos los agentes implicados en el proceso. Los anunciantes podrán comprar las impresiones que les interesan en el mismo momento en que un usuario está visitando la web, decidiendo en tiempo real si se considera público objetivo o no y asegurando su inversión. Los soportes que venden sus espacios cuentan también con la gestión de datos para generar nuevas oportunidades de optimizar sus inventarios, dependiendo de la demanda real existente. El usuario se ve también afectado positivamente: la publicidad será más relevante y de mayor interés para él, resolviendo el desinterés por la excesiva oferta de anuncios que interesan a pocas personas. (p. 200) 
Sin embargo, hay un sector de la doctrina que considera perjudicial la existencia de proveedores que hacen uso de data para concurrir en el mercado, toda vez que, al tener la capacidad de monetizar sus servicios de manera efectiva mediante el uso de publicidad programática o dirigida, generan importantes rentas para los anunciantes a costa de la exposición a la publicidad de los consumidores. Así, señalan que podrían existir ciertos factores que creen "consumidores vulnerables" frente a la publicidad programática que deberían generar una alerta de probable intervención por parte del Estado. El fundamento de ello es que por la gran información que pueden tener los anunciantes acerca de nuestro comportamiento e intereses podría generarse condiciones perjudiciales para los consumidores, como actos de discriminación de precios, a través del cual un mismo producto se venda a precios distintos a dos consumidores sustentado en su capacidad económica o incluso origen geográfico y racial, lo cual también podría generar discriminación de acceso a determinadas ofertas. Asimismo, se sostiene que podrían existir consumidores que sufran de problemas de ansiedad que podrían verse más influenciados por estrategias publicitarias que se realicen sobre la base de sus debilidades, con conocimiento previo de ello (Townley et al., 2017).

\section{PUBLICIDAD PROGRAMÁTICA CONTEXTUAL Y COMPORTAMENTAL O CONDUCTUAL}

De un Windows que contaba con todo el poder de mercado en el uso de plataformas informáticas a través de computadoras y laptops, hemos pasado a dispositivos que son mucho más útiles para los consumidores como los smartpones y tablets (que hacen uso del sistema operativo Android o IOS) por su simplicidad. El gran cambio en el "timón de poder" de las plataformas informáticas se ha presentado en el poder de mercado que tienen ahora Google, Facebook, Instagram entre otras plataformas digitales, las cuales brindan servicios a los consumidores sin que ellos tengan que pagar un monto de dinero por el uso de estos. Como para nadie es un misterio de que no hay almuerzo gratis, dichos servicios se pagan a través de la exposición del consumidor a la publicidad comercial. Y una infraestructura basada en la publicidad tiene su único límite en la cantidad de publicidad que se pueda crear y difundir. Es en este punto donde Google y las redes sociales pueden explotar sin límites la difusión de publicidad, sobre todo Google, por la cantidad de servicios adicionales que pone a disposición a los consumidores, en los que cada uno de ellos puede llegar a ser una plataforma publicitaria (Picker, 2009). Ciertamente, los anunciantes tienen muchas más opciones para difundir publicidad a través de las plataformas que forman parte de Google (entre otros, Google Chrome, Google+, YouTube, Gmail, Android, Google Drive, Google Maps y Google Docs), así como también Google tiene muchas más fuentes de datos de nosotros que cualquier red social. Son diversos los tipos de publicidad que vende Google, por lo que solo vamos a pronunciarnos sobre la publicidad programática.

Diversas plataformas digitales ofrecen servicios online a costo cero (o muy bajo costo), porque su ganancia realmente se encuentra en los ingresos por publicidad. 
Dichos ingresos por publicidad serán rentables solo si su servicio genera una importante demanda en el mercado, por lo que tienen fundados incentivos para invertir constantemente en la mejora de la calidad de su servicio a fin de generar la mayor cantidad de usuarios que bagan uso de su plataforma. La fórmula es simple: mientras más ingresos publicitarios pueda tener un proveedor online de cada usuario, mayores serán los beneficios de atraer y retener usuarios. Ello genera un beneficio directo a los consumidores que tienen todos los días más oferta de plataformas online y servicios que se encuentran en constante evolución en términos de calidad (Lerner, 2014).

Como hemos señalado párrafos atrás, la efectividad de la publicidad comercial se ha visto incrementada gracias al uso de los datos que compartimos con las diversas plataformas digitales que usamos y la que generamos en nuestro usual comportamiento en la web. No cabe duda de que un anuncio es mucho más efectivo si va dirigido a nuestros particulares intereses (Garrido et al., 2018). En este contexto, plataformas como Google y Facebook cuentan con una gran cantidad de data de cada uno de sus usuarios y puede segmentar nuestros perfiles según nuestros gustos, intereses, edad, zonas geográficas y comportamiento en sus respectivas plataformas para poder diseñar espacios publicitarios dirigidos. Asimismo, a través de dichas plataformas, los anunciantes también tienen ahora herramientas para poder enviarnos publicidad de acuerdo con nuestros intereses y comportamiento online (Picker, 2009).

Ahora bien, la publicidad programática tiene dos formas de realizar publicidad dirigida: por contexto y por comportamiento. Sin embargo, al final del día estas estrategias buscan lo mismo: segmentar grupos de perfiles para el envío de publicidad. Lo único que los diferencia es la forma cómo va a realizar tal segmentación, toda vez que ni siquiera son estrategias excluyentes. Muchos anunciantes utilizan ambas para llegar a potenciales consumidores potencialmente interesados en sus ofertas.

En la publicidad dirigida por contexto, el anunciante busca difundir publicidad de acuerdo con lo que demanda un consumidor en determinado momento a través de su navegación en la Internet, sin analizar su comportamiento en la Web (búsquedas realizadas en Yahoo, por ejemplo). Esta estrategia solo busca difundir publicidad en espacios determinados, asumiendo (por estudio de perfiles) que quienes demanden dicha información tienen gustos particulares. Así, la difusión de la publicidad se basa en el lugar donde se encuentre un consumidor en determinado momento. De este modo, si nos concentramos en un diario digital, la publicidad programática por contexto presentará publicidad de corbatas o vestidos formales en la sección de noticias políticas o legales, publicidad de máquinas para hacer ejercicio o ropa deportiva en la sección deportes, maquillaje y cosméticos en la sección moda, etc. Es decir, la publicidad se difunde por perfiles predeterminados, los cuales, evidentemente, no son exactos, pues se guían más por estereotipos definidos por la sociedad. Por su parte, la publicidad programática conductual o comportamental concentra sus criterios de difusión en las acciones que realice el consumidor en su navegación en la Web. Así, si un consumidor realiza una búsqueda de vuelos para Cusco, esta persona puede recibir publicidad sobre hoteles, rentas de auto y atractivos generales que se pueden 
realizar en dicha localidad, pero, además, si el mismo consumidor realizó búsquedas previamente sobre comida vegana y contrató deportes de aventura en un anterior viaje, también recibirá publicidad de restaurantes veganos en Cusco y oferta sobre deportes de aventura de la localidad. Por ello se dice que el "santo grial" de la publicidad programática comportamental es combinar un dato como puede ser la compra potencial con otros factores que se manifiesten de las búsquedas o navegación online previa del consumidor (Tushnet y Goldman, 2014).

\section{Publicidad programática por contexto: publicidad a través de keywords}

A diferencia de la publicidad comportamental o conductual, en la publicidad programática por contexto, los anunciantes segmentan a los usuarios por el contexto de sus visitas. Estos anuncios tienen por objeto llegar a los consumidores en un momento que consideran "propicio", ya que el usuario se encuentra buscando activamente o visitando una página web presumiblemente relacionada con sus intereses actuales. Así, si un usuario se encuentra en una página de autos de determinada marca, es muy probable que se le muestre publicidad de repuestos automotrices, seguros para automóviles o talleres mecánicos. Es decir, se le envía publicidad presumiendo que esta puede estar relacionada con los temas que le interesan. Dicha publicidad llega, sin distinción, a todas las personas que visiten dicha página web (Pérez, 2012).

Posiblemente la estrategia más efectiva de la publicidad programática por contexto sea la que hace uso de keywords o palabras clave. Los anunciantes contratan este servicio para mostrar su publicidad a aquellos usuarios que, en un determinado momento, se encuentran realizando una búsqueda específica a través de un motor de búsqueda (Google, Yahoo, Bing, etc.). En estos casos, la publicidad va a depender de los keywords o palabras clave utilizados por el usuario en su búsqueda concreta. Así, se le mostrará publicidad en los resultados de la búsqueda, la cual se encontrará estrechamente relacionada con las palabras clave usadas. El buscador por su parte procesará la información de dicha búsqueda y puede instalar cookies para almacenar las preferencias de búsqueda del usuario (Pérez, 2012); sin embargo, es importante recalcar que este tipo de publicidad se concentra en el uso de palabras clave, no del comportamiento del usuario a través de la web.

Google Ads es la plataforma de venta de publicidad de Google. A través de dicha plataforma, los anunciantes buscan dirigir su publicidad a un determinado público específico, los que consideren que son los más interesados en sus ofertas. Dicho sistema se configura sobre la base de uso de keywords o palabras clave y cookies. Los anunciantes, en la configuración de su campaña online, compran los keywords con los que creen que los usuarios van a buscar los productos que ofrecen al mercado y pueden diseñar sus campañas para que su publicidad se difunda junto con los resultados orgánicos de una búsqueda en Google, en banners, durante un vídeo de Youtube, o en páginas web relacionadas con las keywords elegidas. El límite de pago por publicidad lo establece el anunciante a modo de subasta en tiempo real y se cobra por clics que los usuarios 
realicen al anuncio digital (y en Youtube por anuncio visto hasta el final). Sin embargo, el tema no es tan sencillo, no se va a difundir de forma destacada el anuncio que pague más, sino el que, en virtud del algoritmo, tenga la mejor configuración de su Ad Rank (que es una evaluación que hace Google por cantidad de visitas que puede generar, relevancia y página de destino) (Sosa, 2020).

Así, se puja por las palabras clave en tiempo real y el algoritmo de Google establecerá los resultados de acuerdo con factores como la relevancia de la palabra clave con la búsqueda (quality score), el page rank de la página (nota de calidad, que se determina si el usuario puede encontrar exactamente lo que está buscando o si la página carga rápido o no, por dar unos ejemplos) y otros como el CTR (cantidad de visitas que ha recibido la web). Es en virtud de dichos factores que Google determina si es o no un buen anunciante y premia o penaliza el anuncio que se pretende difundir (Cyberclick Marketing Digital, 2020).

Una de las características particulares de la publicidad a través de Google Ads es que se trata de un sistema de publicidad de pago por clic. Es decir, se le paga a Google por la cantidad de veces que el usuario le da clic al anuncio y es el anunciante es el que establece cuánto es el máximo que está dispuesto a pagar por clic. Ello permite que los anunciantes puedan cuantificar su inversión de una manera más exacta que con los medios tradicionales de comunicación publicitaria, toda vez que puede tener información de las veces que los consumidores dieron clic en su página web producto de la publicidad e incluso poder relacionar dicha información con la cantidad de ventas online que pueda generar (Fletcher, 2010, p. 96).

Es importante no confundir la forma en la que se obtienen los resultados orgánicos (no patrocinado) de los publicitarios (patrocinados) en los motores de búsqueda. El contenido no patrocinado de la página de resultados de búsqueda proviene de un algoritmo que intenta ofrecer los resultados más relevantes en función de las palabras clave que el consumidor ha escrito y va a llegar a ellos a través de los metatags (información contenida en el código fuente de la página web sobre, entre otras cosas, contenido, idioma, ubicación geográfica, adaptación de la web a dispositivo con el que se hace la búsqueda, etc.) y relevancia del contenido de la página web (Sosa, 2020). Por su parte, el contenido patrocinado de la página de resultados de búsqueda proviene de un algoritmo diferente que tiene en cuenta tanto la relevancia del anuncio publicitario para la consulta del usuario como la cantidad máxima que un anunciante está dispuesto a pagar por palabras clave específicas, entre otros factores antes comentados. El algoritmo determina cuántos anuncios colocar en la página de resultados de búsqueda y dónde colocarlos (Mariscal y Evans, 2012, p. 4).

La controversia legal sobre esta estrategia publicitaria recae en que algunos agentes económicos, con el objeto de posicionar la publicidad de su oferta en las búsquedas que más realizan los consumidores, compran como palabras clave o keywords las marcas de sus competidores si estas cuentan con mayor implantación o demanda en el mercado. Ciertamente, este tipo de publicidad hace uso de algoritmos y no pone restricciones ni límites al uso de palabras clave o keywords, pues, finalmente, no es el único elemento 
de relevancia con el que el algoritmo funciona; sin embargo, no son pocos los casos en los que se han comprado como palabras clave marcas que gozan de gran reputación o demanda por parte de los consumidores, sin autorización de sus respectivos titulares.

\section{Publicidad programática comportamental o conductual}

Conforme a lo adelantado, en este tipo de publicidad, la publicidad se difunde a determinado grupo de consumidores en virtud de la segmentación que se pueda realizar por su comportamiento online. El punto sensible y controvertido de este tipo de publicidad, es entender la forma como los anunciantes o plataformas digitales pueden obtener la información de navegación o los intereses específicos de los consumidores desplegados con su comportamiento en internet. Los datos se pueden obtener de propia voluntad del consumidor, como es el registro voluntario del usuario o consumidor en las bases de datos de determinadas empresas o aplicaciones, como la información de nuestros intereses que le otorgamos a una red social y nuestro desenvolvimiento en la misma; sin embargo, los datos también se pueden obtener producto del uso de técnicas electrónicas sin la autorización ni el conocimiento de los usuarios (López, 2013).

En el primer caso, estamos frente a nuestros intereses, gustos y preferencias que le proporcionamos voluntariamente a las redes sociales de las que formamos parte. Por el lado de Facebook e Instagram, desde nuestro registro en dichas redes sociales, le hemos compartido nuestra información de perfil y día a día le compartimos información sobre nuestros intereses y gustos (desde que seguimos una tienda de ropa o los restaurantes que visitamos). La gran mayoría de usuarios de dichas redes sociales (ambas manejadas por Facebook) comparten información fidedigna y ello permite que Facebook sienta seguridad sobre los perfiles que maneja (a pesar de que obviamente, existen cuentas falsas). De esta forma, Facebook puede segmentar nuestros perfiles con mucha facilidad, lo cual le permite ser un medio de comunicación de publicidad programática para empresas interesadas en difundir publicidad a perfiles específicos (la plataforma de venta de publicidad a través de esta red social es Facebook Ads). Sobre la capacidad de impacto publicitario de las redes sociales, Touriño (2014) señala que:

Las redes sociales y también otras muchas plataformas de Internet saben más de nosotros que muchas de las personas de nuestro propio entorno. Si pensamos, por ejemplo, en una red social a la que accedamos de manera asidua, podremos comprobar que con el mero registro en la red aportamos a dicha red datos sobre nuestra edad, nuestra ubicación geográfica, el lugar en el que hemos estudiado o en el que trabajamos. Ello permite ya a la red social crear un perfil sociocultural de usuario que se registra. Pero, además, si el usuario va alimentando su perfil y compartiendo datos e información sobre, por ejemplo, sus viajes, los grupos de música que sigue o los libros que lee, la red social estará en posición en tal caso de crear un auténtico perfil de las preferencias y los gustos del usuario. Es por ello que cuantos más datos tenga de nosotros una red social, más interesante y rica será para el resto de usuarios de la propia red, pero también para quienes pretendan 
promocionarse en ella, por cuanto que el grado de segmentación del público al que dirigirse es mayor y la publicidad, al menos en potencia, más efectiva [...] En definitiva, ese volumen de información que sí posee Facebook permite a la red social más populosa del planeta ofrecer bases de datos a empresas interesadas en prospecciones de mercado o en negocios basados en perfiles personales. Se posibilita así investigar las preferencias de un segmento de la población, acotado según edades y área geográfica y llegar al público adecuado en el momento justo. (pp. 48-49)

Facebook (2020) detalla en la aplicación cómo utiliza nuestros datos para poder difundirnos publicidad comercial de terceros manteniendo la privacidad de nuestros datos personales, los cuales en ningún supuesto se comparten con las empresas anunciantes —al menos es lo que nos dice su propia aplicación-. Así, tenemos que los anuncios que nos llegan a través de dicha plataforma o en otras páginas web son producto de

- Nuestra actividad en empresas y productos de Facebook: para difundirnos estos anuncios, dicha aplicación hace uso de la información que le hemos compartido al momento de indicarle las páginas que nos gustan, nuestra información de perfil que hemos llenado y lugares que hemos visitado. Podemos obtener dicha información y, además, controlarla, si nos dirigimos a la sección "configuración" y editamos las preferencias de anuncios que le hemos compartido a la aplicación desde que nos suscribimos.

- Nuestra actividad con otras empresas: muchas veces compartimos nuestra información voluntariamente con una determinada empresa, como pueden ser nuestros datos de correo electrónico o número de celular. Ello puede suceder cuando nos registramos en un diario digital o blog para recibir un boletín de noticias por correo electrónico, cuando hacemos compras online en determinadas tiendas, cuando nos registramos para recibir cupones o descuentos. Dichas empresas, muchas veces hacen publicidad en Facebook y añade sus datos a una lista de clientes de potenciales clientes que Facebook pone a su disposición; sin embargo, no tiene un acceso directo a dichos datos personales. Esa información, una vez que es ingresada, llega encriptada a Facebook y es dicha aplicación la que a través de su algoritmo busca coincidencias con la información codificada que tienen del perfil de sus usuarios. Si existe una coincidencia, se le envía la publicidad a dicho perfil.

- Nuestra actividad en otros sitios web y aplicaciones: Facebook pone a disposición de las empresas "las herramientas de Facebook para empresas" que permiten integrar la actividad de sus usuarios con Facebook a través de diversas herramientas. Una de estas herramientas, que es la más común, es la que permite que puedan iniciar una sesión de una aplicación con Facebook. Cuando un usuario abre una determinada plataforma online con su sesión de Facebook está compartiendo datos con dicha plataforma, lo cual va a permitir que se le pueda enviar publicidad basada en los productos o servicios que ha consultado en 
dicha página web. Esto también sucede usualmente cuando descargamos una aplicación y la abrimos con nuestra sesión de Facebook.

- Nuestra ubicación: Facebook utiliza la información de localización que la trasladamos a la aplicación, por ejemplo, desde el lugar donde nos conectamos, los lugares donde usamos el smartphone o la ubicación que hemos compartido en nuestro perfil de Facebook o Instagram. De esta forma, utiliza nuestra información de ubicación para enviarnos publicidad de anunciantes que intentan llegar a potenciales consumidores de un lugar específico.

Cabe señalar que Facebook brinda a sus usuarios la posibilidad de controlar y decidir los anuncios que quiere ver ocultándolos desde el propio anuncio (dándole clic a los tres puntos que figuran al lado derecho del post publicitario, por ejemplo) u organizando sus preferencias de anuncios en la sección correspondiente a la que puede acceder de forma muy sencilla en "configuración".

Ahora bien, con toda esta información que Facebook tiene a su disposición es que se produce la "magia" de Facebook Ads. Los anunciantes que pretenden difundir su publicidad a un determinado público dirigido indica a Facebook la audiencia deseada (en función a lo que crea que es el público a quienes más les interesará su oferta) y le comparte el anuncio que pretenda que se difunda, indicando las plataformas en las que quiere que se realice tal difusión (Facebook, Instagram, sitios web y aplicaciones móviles), los cuales sube con las herramientas de administración de anuncios que la aplicación pone a su disposición. Finalmente, con dicha información, Facebook comparte el anuncio a las personas que cumplan con el público objetivo determinado por el anunciante sin compartirle a dicha empresa los datos de los perfiles a los que se les envía la publicidad. Así, Facebook nos envía publicidad acorde a nuestros intereses compartidos y permite que los anunciantes puedan dirigir sus anuncios a verdaderos potenciales compradores, lo cual, en la mayoría de los casos representa la efectividad de la publicidad dirigida a través de redes sociales como Facebook e Instagram (Tucker, 2016).

De este modo, nos debe quedar claro que Facebook no cede nuestros datos personales a terceros anunciantes, todo lo contrario, utiliza toda esa información que nosotros le hemos otorgado voluntariamente para ofrecer un servicio de publicidad segmentado, es decir, funciona como un medio de difusión de publicidad dirigida al tipo de perfil que sea el objetivo del anunciante.

De este modo, el fabricante de zapatos de fiesta para mujeres puede solicitar al titular de la plataforma [...] que únicamente dirija su publicidad a su público específico. Así, en lugar de malograr el coste derivado del envío de publicidad a destinatarios que para nada están interesados en su oferta, tal y como sucedía con las plataformas tradicionales, estos nuevos medios permiten segmentar el mercado, decidir a quién queremos dirigir la publicidad de nuestros productos o servicios. 
O por decirlo de otro modo, la marquesina del autobús que aloja publicidad, o el periódico generalista en papel, tiene el coste superfluo de individuos no interesados en el producto o servicio que ofertamos. Sin embargo, la red social puede ofrecer al anunciante el dirigir su anuncio, por ejemplo, a mujeres entre 20 y 35 años que residan en un determinado lugar y tengan un determinado perfil socioeconómico. Lo que es más, pueden decidir que solo se ofrezca su producto a quienes le gusten los zapatos de fiesta de la competencia. Esto es tan sencillo para una red social como Facebook como identificar los "me gusta" que ha hecho cada usuario. Así una marca podrá, precia remuneración a Facebook, pedir a esta que dirija sus promociones únicamente a quien alguna vez haya pulsado "me gusta" en algún producto similar al suyo. (Touriño, 2014, pp. 50-51)

Este tipo de publicidad programática comportamental (a través de redes sociales) es una de las más eficaces en la medida que, por lo general, compartimos muchas cosas de las que hacemos todos los días por las redes sociales, así como también manifestamos diariamente nuestros intereses por determinado contenido a través de estas. Todo ello, lo hacemos con el claro conocimiento de que esa información la estamos compartiendo con nuestros contactos y la red social, por lo que si por ahí tenemos a un consumidor despistado, que pensó que la red social no iba a sacar algún tipo de beneficio con la información que públicamente comparte, lo cierto es que desde hace algunos años Facebook, Instagram y Twitter, por mencionar algunos, nos informan cómo se nos envía publicidad programática de acuerdo con nuestros intereses y gustos compartidos voluntariamente con sus plataformas.

Sin embargo, la situación se complica cuando nos ubicamos en la publicidad programática comportamental que llega a nosotros a través de datos que no compartimos voluntariamente. Gran parte de la publicidad programática comportamental llega a nosotros gracias al seguimiento que hacen de nuestro comportamiento online las cookies de búsqueda tracking cookies. De hecho, es importante precisar que, para cierto sector de la doctrina, la publicidad comercial que se nos difunde a través de Facebook Ads es solo publicidad social (social advertising) y no se trata de publicidad programática comportamental, toda vez que nosotros hemos compartido voluntariamente nuestra información de perfil e intereses con la red (Pérez, 2012). Sin embargo, como hemos podido ver de la forma como funciona Facebook Ads, si bien se trata de publicidad que nos llega según la información que de forma voluntaria le brindamos a la red social, lo cierto es que también nos envía publicidad de acuerdo con nuestro comportamiento, cuando abrimos o nos registramos en plataformas o aplicaciones a través de Facebook o incluso cuando nos envía publicidad de acuerdo a los lugares donde nos etiquetamos o la zona donde geográfica específica desde donde utilizamos el smartphone.

Ahora bien, para definir las cookies, empezaremos por la parte más básica. Las cookies son pequeños ficheros de textos que se nos puede instalar en la computadora al visitar una página web. Este archivo servirá para condensar información técnica y útil de nuestra visita en dicha página, como, por ejemplo, el idioma que hemos seleccionado, la zona geográfica en la que nos encontramos, la información de nuestro 
carrito de compra, nuestro usuario y contraseña, entre otros (se puede archivar todo tipo de información en una cookie, incluso todas las visitas que realicemos en nuestra navegación online). Así, cada vez que volvamos a visitar dicha página web, ya no será necesario que volvamos a seleccionar idioma, poner nuestro nombre o indicar desde qué parte del mundo estamos ingresando. Esa información es leída de la cookie que tenemos instalada en nuestra computadora y es utilizada por la página web que nos la instaló. De este modo, las cookies permiten conseguir información sobre los hábitos de navegación de los consumidores (López, 2013). Así, sobre la base del funcionamiento descrito, Touriño (2014) señala que

cuando el usuario vuelve a visitar la página que le instaló la cookie en su navegador este sabrá qué noticias ha leído, qué canciones ha escuchado en Spotify o en qué redes sociales ha estado compartiendo información.

Las cookies permiten a las empresas gestionar de forma eficaz sus espacios publicitarios y hace un seguimiento de la navegación del usuario porque conocen precisamente dónde va el usuario. Las cookies permiten ofrecer campañas publicitarias en función de la información que recolectan, que no es otra que la estela que dejamos al navegar. (p. 58)

En efecto, los desarrolladores web desde hace un tiempo se han dado cuenta que la información que se puede almacenar en las cookies pueden ser utilizadas con fines publicitarios, por lo que ahora, muchas de estas cookies se instalan solamente con un código de identificación y el resto de la información se encuentra en los propios sistemas de las páginas web, permitiendo que pueda almacenar toda tu información sin limitación alguna y que mediante esta identificación, otras páginas puedan identificar la cookie instalada en la computadora y puedan enviarle publicidad comercial de acuerdo a su comportamiento online, donde toma vital importancia las tracking cookies que se nos instalan cuando visitamos una página web y sirve para segmentar nuestra información de comportamiento online en todas las páginas web que conformen una red publicitaria (López, 2013).

De esta forma, se puede segmentar perfiles y ubicarlos en distintos grupos a los cuales se les pueda dirigir una concreta publicidad. De ello se encarga un editor web o una red publicitaria, los que ponen dichas audiencias a disposición de los anunciantes que estén interesados de dirigir su publicidad a perfiles que coincidan con los segmentados. Por ejemplo, a los usuarios que hacen búsquedas de vuelos y turismo en determinadas páginas, se le incluirá en un tipo de perfil de "viajeros", pues queda claro que tiene un potencial intereses en dicha oferta y ello va a permitir que una aerolínea o empresa de turismo pueda enviarle publicidad la próxima vez que acceda a internet, independientemente de la página web que se encuentre visitando en ese momento. Ciertamente, es importante precisar que, si bien este tipo de cookies no tiene como objetivo un eventual tratamiento de datos personales, uno de los principales cuestionamientos que enfrenta el uso de esta estrategia publicitaria es que se afectaría 
a la privacidad de los usuarios, quienes, por lo general no tendrían conocimiento que se está monitorizando su comportamiento online (Pérez, 2012).

Con todo lo anterior, podemos señalar que la publicidad programática comportamental es la que se hace efectiva a través del uso de cookies que generan información del comportamiento en la Web. Sin embargo, es importante precisar que la publicidad comportamental no se ciñe solo a ser un anuncio basado en la conducta del consumidor. De acuerdo con Pérez (2012), estamos frente a una estrategia publicitaria que utiliza técnicas de segmentación a través del rastreo de la actividad llevada a cabo por los navegadores, la cual se lleva a cabo por tracking cookies instaladas en los terminales. Así, esta técnica publicitaria implica el análisis de hábitos de comportamiento y navegación online, lo cual implica búsquedas de palabras clave realizadas en motores de búsqueda, la frecuencia de visitas a webs concretas, los clics que se han realizado a determinada publicidad o link de información, tiempo de permanencia en ciertos contenidos, etc. Evidentemente, no estamos hablando de un usuario específico, la publicidad que se envía a través de esta estrategia va dirigida a un concreto navegador instalado en un terminal electrónico que, evidentemente, es utilizado por un usuario durante su navegación.

[Así, ] podemos definir la publicidad comportamental como aquella publicidad que se muestra durante una concreta navegación, por razón de la actividad online que se ha venido desarrollando durante un periodo de tiempo determinado, desde ese mismo navegador. (Pérez, 2012, pp. 13-14)

\section{¿ES NECESARIA LA REGULACIÓN DE LA PUBLICIDAD PROGRAMÁTICA?}

En el presente apartado comentaremos algunos de los criterios más importantes que se han ido desarrollando a nivel europeo y norteamericano sobre la publicidad programática contextual a través de keywords y sobre la publicidad comportamental o conductual.

\section{Publicidad contextual a través de keywords}

La discusión a nivel de la jurisprudencia europea ha dejado varios criterios interesantes en los últimos diez años en diversos casos a través de los cuales se denunciaba infracción marcaria por supuesto uso indebido de la marca de un tercero como keyword sin autorización de su titular. Cabe señalar que a nivel europeo no hay disposición específica que regule la publicidad programática contextual con el uso de keywords a través de motores de búsqueda. Los aspectos controvertidos que más se han discutido sobre el uso de esta estrategia publicitaria se han concentrado en infracciones marcarias y de competencia desleal. Así, por ejemplo, para los autores Martínez y Miralles (2014), el hecho de que un operador económico haga uso de marcas de terceros como palabras clave para contratar publicidad en motores de búsqueda constituye un acto 
de competencia desleal, toda vez que dicho agente a través de esta estrategia, "pretende que personas que a través del buscador se dirigían directamente a la web del anunciante, lo hagan pasando por su enlace o por su web, atribuyéndose así de forma desleal el mérito de estas visitas" (p. 76).

Por mencionar algunos casos relevantes, el Tribunal de Justicia de la Unión Europea (TJUE) señaló mediante sentencia en el asunto C324/09, del 12 de julio de 2011, en el caso L'Oréal vs. eBay, que el titular de una marca está facultado para prohibir a un tercero hacer publicidad, a partir de una palabra clave idéntica a esa marca, cuando dicha publicidad no permita o apenas permita al consumidor determinar si tales productos proceden del titular de la marca o de una empresa económicamente vinculada a este o si, por el contrario, proceden de un tercero. En el referido caso, L'Oréal denunció, entre otras cosas, que eBay era responsable del uso de marcas de L'Oréal como consecuencia de la presentación de estas en su sitio web y de la inserción en el sitio web de operadores de motores de búsqueda como Google de enlaces patrocinados que aparecían cuando se empleaban palabras clave correspondientes a dichas marcas. Así, de acuerdo con la denuncia, eBay, mediante la compra de palabras clave correspondientes a marcas de L'Oréal, generaba la aparición, en los resultados de búsqueda, de un enlace publicitario al sitio www. ebay.co.uk cada vez que un usuario buscaba productos L'Oréal.

Posteriormente, el TJUE hizo un análisis más profundo sobre el particular en el Asunto C323/09, en la interpretación prejudicial contenida en su sentencia del 22 de septiembre del 2011, en el que reiteró que el uso de marcas de terceros como palabras clave será ilícito cuando afecte la función de origen empresarial de la marca (es decir solo cuando exista riesgo de confusión); sin embargo, destaca que el uso de esta estrategia publicitaria puede hacer la competencia más eficiente entre agentes económicos y generar más información a los consumidores, quienes tendrán más alternativas para tomar una decisión de consumo. Así, sostiene el referido tribunal que el uso de la marca de un tercero en Google Ads sin autorización de su titular no afectará la función publicitaria de la marca: "la elección de un signo idéntico a la marca de un tercero [...], no priva al titular de dicha marca de la posibilidad de utilizar eficazmente su marca para informar y persuadir a los consumidores".

Todos los criterios anteriores fueron recogido por los tribunales españoles que, por ejemplo, en la Sentencia en Casación n. ${ }^{\circ}$ 105/2016, del 26 de febrero de 2016, estableció que la empresa Charlet S.A.M. hizo uso lícito de las denominaciones MASALTOS y MASALTOS.COM como palabras clave en Google Ads, a pesar de que coincidían con el elemento denominativo de las marcas de Maherlo Ibérica, S.L. que había sido la empresa pionera en introducir los zapatos de marca mixta MASALTOS que tenían como particularidad hacer crecer unos 3 centímetros de estatura gracias a una plantilla especial. Charlet había utilizado los elementos denominativos antes mencionados como keywords a fin de posicionar en los resultados de Google sus zapatos que podían hacer crecer hasta siete centímetros de estatura a las personas que lo usen. Así, en la referida sentencia, se señaló que 
dado que el derecho de exclusiva no es absoluto, tal uso únicamente será considerado infracción de marca cuando el uso de la marca ajena se haga a título de marca, es decir con el fin de identificar un determinado producto o servicio. Es decir, se pueden utilizar marcas registradas como palabras clave para mostrar enlaces patrocinados siempre y cuando se cumplan con una serie de requisitos: (i) que el uso de la marca no menoscabe ni la función indicadora del origen de la marca, ni su función económica, (ii) que resulte claro para un usuario medio de internet que los productos o servicios publicitados no proceden del titular de la marca o de una empresa económicamente vinculada; y de no ser así, se indique bajo qué circunstancia se venden productos de una determinada marca a través de una página web distinta a la "oficial". La finalidad de este requisito es impedir el riesgo de confusión [...].

Por otro lado, en Estados Unidos no hay una posición uniforme sobre si el uso de marcas de terceros en motores de búsqueda se trata de un acto ilícito o n. ${ }^{\circ}$ Se ha planteado la posibilidad de sancionar este tipo de actos por la doctrina de la "Confusión en el interés inicial del consumidor", la cual se presentaría en la inducción a error inicial que podría generarse a los consumidores en los resultados de sus búsquedas al ver que salen como resultados patrocinados productos similares o marcas competidoras. De acuerdo con Amunátegui (2006), la doctrina de la "Confusión en el interés inicial del consumidor" consiste en considerar que el uso de marcas de terceros — sin autorización de su titular - que provoca como consecuencia que figuren resultados patrocinados junto a la oferta que estaba buscando el usuario de un motor de búsqueda, producen una confusión inicial en los usuarios, toda vez que al encontrar diferentes páginas en los resultados de la búsqueda, el consumidor no sabe exactamente cuál de ellos corresponde efectivamente a la empresa u oferta que busca.

Aunque con un somero análisis el usuario dará con la página que se proponía encontrar, lo cual hace bastante cuestionable considerar que podría existir un riesgo de confusión "inicial", lo cierto es que el operador económico que utilice esta estrategia va a poder ubicar la publicidad de su marca junto con la página de su competidor. Así, se entiende que las empresas competidoras, en su afán de acceder al mercado, se están "colgando" del buen nombre de la empresa que genera una mayor demanda en el mercado. De ahí proviene el origen del reproche de la lealtad que la jurisprudencia norteamericana asigna a tal conducta, puesto que en base a la reputación y buen nombre de una empresa sus competidores, ahorrándose costos de posicionamiento y concurrencia en el mercado, se aprovechan deslealmente de ello utilizando sus marcas para posicionar sus ofertas (p. 253).

Según Fueroghne (2007), el origen de dicha teoría o doctrina terminó de desarrollarse en el caso "Brookfield Comunications, Inc vs. West Coast Entretainment Corporation", que enfrentó a estas dos compañías de la industria cinematográfica, toda vez que West Coast había consignado indebidamente la marca "moviebuff" (perteneciente a Brookfield) como metatag en el código fuente de su página web. La corte explicó la infracción cometida por Wet Coast de la siguiente manera: 
Supongamos que un competidor de Wets Coast (Blockbuster por ejemplo), coloca un panel publicitario en el que se puede leer desde la carretera lo siguiente "West Coast Video: 2 millas más adelante en la Salida n. ${ }^{\circ} 7^{\prime \prime}$, pero West Coast realmente está ubicado en la Salida n. 8 y Blockbuster está ubicado en la Salida 7. Los consumidores que estén buscando la tienda de West Coast saldrán por la Salida n. ${ }^{\circ}$ y manejarán buscando por esta. Incapaces de ubicar la tienda de West Coast, pero viendo la tienda de Blockbuster al lado derecho de la entrada a la carretera, podrán simplemente alquilar en esta última. (pp. 505-506)

Dicha figura ha merecido varias críticas de la doctrina más especializada que considera que establecer límites marcarios o de competencia desleal a esta estrategia publicitaria solo la limitaría y evitaría la evolución de la publicidad comercial online, desconociendo todos los efectos positivos que genera en el mercado, como es el permitir a los consumidores poder comparar entre las ofertas disponibles que obtiene de sus búsquedas en Google, Bing o Yahoo (Klein y Glazer, 2003).

Se sostiene que esta doctrina desconoce que los usuarios saben cómo navegar por internet y pueden reconocer con facilidad un enlace patrocinado de uno orgánico. Esta protección excesiva de los derechos de exclusiva del titular de una marca puede terminar afectando directamente a los consumidores al reducirle fuentes de información (Zweihorn, 2006). Por su parte, Lastowka (2007) señala que la doctrina de la "Confusión en el interés inicial del consumidor" puede incrementar los costos de búsqueda de información del consumidor a través de motores de búsqueda que son realmente útiles en su toma de decisiones de consumo. A decir de dicho autor, esta teoría, más que convencernos de los beneficios a los consumidores, en su lugar, nos deja claro los perjuicios que podría generarles, toda vez que esta teoría es una amenaza al acceso a la información al propiciar incentivos perversos para que los titulares de marcas traten de monopolizar palabras si estas coinciden con sus marcas, por lo que debemos rechazar cualquier tipo de doctrina que pretenda incrementar de forma desmedida los derechos de exclusiva que un titular puede tener sobre determinado término. Por otro lado, Otamendi (2012) señala que la publicidad programática a través de keywords o palabras clave no es más que un mecanismo que permite una más rápida y mejor exposición del sitio que se pretende promocionar en las páginas de internet, por lo que en principio no la considera ilegal y desarrolla el siguiente ejemplo para demostrar ello:

Imaginemos que una firma le da instrucciones a una agencia de publicidad de que cada vez que vea un cartel con la marca X en determinados lugares, coloque otro con la marca Y. Estas instrucciones serán dadas por escrito y, por ende, llevarán impresa la marca X. Más allá de la mecánica y el medio en que se hará la publicidad, estamos frente a un caso igual al aquí analizado. Se trata de estar presente, con publicidad paga o simplemente con el sitio en una página, y cerca de las marcas líderes. Obviamente este hecho no tiene nada de ilegal y es lo que se conoce como competencia. Si no es ilegal el estar cerca del 
competidor en la vía pública o en un programa de televisión, en un estadio o en los avisos de un diario, tampoco lo es si el ámbito es Internet. (pp. 6-7)

De este modo, en línea con lo señalado por Mariscal y Evans (2012), consideramos que las autoridades encargadas de pronunciarse sobre este tipo de actos, antes de resolver de forma restrictiva, deberá tener en consideración que los efectos de censura de esta modalidad publicitaria podrían generar: (i) pérdida de información al consumidor que, gracias al uso de esta estrategia, tiene más información sobre los productos que demanda en el mercado, pues al aparecer en sus resultados de búsquedas ofertas competidoras, puede comparar beneficios, atributos y precios; y (ii) la reducción de la competencia, toda vez que eliminaría una herramienta que actualmente usan las empresas para identificar e informar a los consumidores que han expresado interés en los productos de sus competidores (pp. 6-14).

Destaquemos que no todos los supuestos de uso de la reputación de la marca ajena son algo negativo para el mercado. La publicidad de naturaleza alusiva y en especial la publicidad comparativa son buenos ejemplos de cómo una estrategia publicitaria se puede aprovechar del posicionamiento de otra para informar acerca de su ventaja en el mercado y finalmente generar un efecto positivo para el mercado y consumidores. En efecto, si la publicidad comparativa es lícita reducirá costos de transacción en los consumidores (tiempo de búsqueda de ofertas) e incrementará la competencia entre agentes económicos, ya que permitirá que un nuevo entrante al mercado o una oferta que no tenga gran posicionamiento se termine comparando con la marca de mayor implantación o demanda en el mercado para comunicar su ventaja competitiva (Sosa, 2018).

Otros ejemplos de uso de marcas o reputación de terceros los podemos encontrar en el pago por posicionamiento de productos en anaqueles de supermercados. En muchos casos un operador económico puede pagar más para que su producto esté ubicado cerca de sus competidores más demandados, o, en otros casos, las estrategias que algunas empresas usan para aprovecharse de la compra de productos de sus competidores. Por ejemplo, que la empresa X pague a un supermercado para que cuando los competidores compren un producto de su competidor, se le entregue junto con el comprobante de pago un vale de descuento para la compra de sus productos. Finalmente, quien se beneficia con esta estrategia es el consumidor al que se le ofrece un producto que demanda por menos precio. Como sucede con las modalidades descritas, el uso de marcas de terceros en keywords puede generar como efecto el incremento de la competencia entre operadores económicos, lo cual siempre va a ser beneficioso para los consumidores, así como también es un mecanismo que permite a las nuevas marcas entrantes poder comunicar su oferta al mercado, lo cual también genera un escenario que beneficia al consumidor al tener información sobre una oferta alternativa a la que normalmente elige (Mariscal y Evans, 2012).

Como vemos, el uso de marcas de terceros en keywords puede producir beneficios para los consumidores en la medida que permite que accedan a más información para 
tomar sus decisiones de consumo al conocer ofertas alternativas a la buscada, lo cual, evidentemente permite que se incremente y se haga más agresiva la competencia, incluso, permitiendo que agentes económicos entrantes puedan reducir su costo de posicionamiento intensificando de este modo la competencia que, como sabemos, siempre beneficia a los consumidores al generar como efecto que los productos tengan más calidad y se vendan a precios menores (Sosa, 2020).

\section{Publicidad comportamental o conductual}

Los gobiernos vienen reaccionando de diversas formas frente a la publicidad comportamental. Por ejemplo, en el caso de Estados Unidos, la Federal Trade Commission (FTC) impulsa a que la propia industria, a través de la autorregulación (sistema privado de fiscalización a través del cual la propia industria se autoimpone códigos de conducta), cree sus propias políticas frente a la publicidad comportamental. De hecho, más del 90\% de la industria publicitaria interactiva de Estados Unidos se encuentra cubierta por la autorregulación. Algunas de las reglas que se imponen a través de la autorregulación a los anunciantes consisten en (i) brindar a los consumidores información clara acerca del recabo y uso de sus datos y formas de desvincularse del uso de sus datos; (ii) uso de medidas de seguridad razonables en la recolección de datos; (iii) obtener el consentimiento de los consumidores sobre cualquier cambio en sus políticas de privacidad ${ }_{i}$ y (iv) requerir el consentimiento de los consumidores para la recolección de información sensible incluyendo información financiera y de salud (Jordan, 2014, pp. 481-482).

En el caso de Australia, en el 2011, su sistema de autorregulación (Australian Digital Advertising Alliance) publicó lineamientos para el uso de la publicidad comportamental, el cual complementa la Ley de Privacidad de dicho país. Los objetivos de los referidos lineamientos se concentran en cuatro puntos principales: (i) promover la transparencia en la información para los consumidores acerca que datos va a recabar, cómo va a recolectar dicha información y para qué la va a usar, permitiendo que los consumidores puedan elegir no recibir publicidad comportamental, (ii) fomentar buenas prácticas en las áreas de privacidad, seguridad de datos y el manejo de datos sensibles; (iii) fomentar conciencia en los consumidores con el lanzamiento de la página www.youronlinechoices.com.au y (iv) fomentar el acceso a los consumidores a que puedan tener procedimientos de reclamos y a realizar un monitoreo continuo de los lineamientos. Ahora bien, en lo que a dispositivos legales se refiere, en el caso de Alemania, las secciones 12(1) y 12(2) de la Telemediengesetz (Ley relativa a determinados servicios de comunicación e información electrónicos) o Telemedia Act disponen que los datos personales pueden usarse para personalizar el envío de publicidad comercial únicamente cuando se cuente con el consentimiento del usuario.

Asimismo, de acuerdo con su sección 15 (3), el proveedor de servicios podrá elaborar perfiles de usuario con seudónimos para fines de publicidad, estudios de mercado y para la configuración personalizada de servicios de comunicación e información 
electrónicos, siempre que el usuario no se oponga a ello después de haber sido informado sobre su derecho de oposición. Cabe añadir que la publicidad comportamental es sancionada como publicidad encubierta de conformidad con lo dispuesto por la sección 4(3) de su Unfair Competition Act. En el caso de Reino Unido, su regulación sobre privacidad online prohíbe el almacenamiento de información o acceso a la información de los usuarios de internet a través de cookies, a no ser que (i) se le brinde información clara y comprensible a los usuarios acerca del propósito de recabo, acceso y uso de su información; y (ii) que el usuario haya brindado su consentimiento. Asimismo, se dispone que los que recaban y usan datos para ejecutar publicidad comportamental tienen que informar claramente en la misma publicidad que se está haciendo uso de esta estrategia publicitaria e incluir mecanismos para que los consumidores puedan elegir que no se recopile su información y, además, exige que no se deben crear segmentos de interés para niños de 12 años o menores para recabar datos a fin de utilizarlos para enviarles publicidad comportamental (Jordan, 2014).

A nivel de la Unión Europea, se regula el uso de datos recolectados por cookies a través del Reglamento 2016/679, que en su considerando 32 dispone, como obligación, que la instalación de cookies debe ser debidamente informada a los usuarios y se debe contar con su consentimiento activo. Dicho consentimiento debe darse mediante un acto afirmativo claro que refleje una manifestación de voluntad libre, específica, informada e inequívoca del interesado de aceptar el tratamiento de datos de carácter personal que le conciernen, como una declaración por escrito, inclusive por medios electrónicos, o una declaración verbal. Es en esa línea que el TJUE, mediante Asunto C673/17 del 1 de octubre de 2019, señaló que dicho consentimiento podría incluir marcar una casilla de un sitio web en internet, escoger parámetros técnicos para la utilización de servicios de la sociedad de la información, o cualquier otra declaración o conducta que indique claramente en este contexto que el interesado acepta la propuesta de tratamiento de sus datos personales. Por tanto, el silencio, las casillas ya marcadas o la inacción no deben constituir de ninguna manera consentimiento. En dicho Asunto, el TJUE absolvió una cuestión prejudicial señalando que el consentimiento de instalación de cookies no se presta de manera válida cuando el almacenamiento de información o el acceso a la información ya almacenada en el equipo terminal del usuario se autoriza mediante una casilla marcada por defecto de la que el usuario debe retirar la marca en caso de que no desee prestar su consentimiento.

Sin perjuicio de lo anterior, hasta el día de la publicación del presente artículo, se discute la legalidad del uso de datos de las plataformas online, lo cual podría haber generado un problema de competencia en la medida que existiría empresas que han logrado un gran poder de mercado por la cantidad de data que manejan de usuarios y no permitirían el ingreso de nuevos operadores económicos (en líneas generales). De hecho, en la Unión Europea se encuentra en debate público una propuesta regulatoria a las plataformas digitales (Comisión Europea, 2020), donde se buscan establecer restricciones para este mercado al que definitivamente se trasladarán dentro de poco la mayor parte de los agentes económicos. Algunos riesgos que se identifican se 
concentran también en los potenciales actos de discriminación en el consumo que podrían generar en perjuicio de los consumidores (Townley et al., 2017).

Sea a través de normas o de la autorregulación, consideramos positivo que las restricciones sean sensatas y se concentren más en exigir traslado de información a los usuarios sobre la instalación de cookies y solicitarles su autorización. En Sudamérica no se debería seguir un camino distinto o exigir algún tipo de censura respecto del uso de la publicidad comportamental o conductual. Tushnet y Goldman (2014) sostienen que cualquier regulación que pretenda prohibir o censurar la publicidad programática comportamental, lo único que generará como consecuencia es que el consumidor siga recibiendo publicidad, solo que ya no de acuerdo con su comportamiento. Así, quien finalmente se perjudica con una eventual prohibición es el consumidor a quien le va a seguir llegando excesiva publicidad, pero menos útil que la que le puede llegar si es dirigida según sus intereses por su comportamiento online. Por su parte, Lerner (2014) sostiene que el tratar de controlar o restringir la forma como se maneja actualmente la publicidad digital programática comportamental solo podría generar como efecto la reducción de la competencia y que los proveedores de servicios en línea empiecen a cobrar precios más altos ajustados a sus servicios en línea (Lerner, 2014).

En ese sentido, Chen y Stallaert (2010) destacan la labor de la autorregulación frente a los riesgos que pueda presentar la publicidad comportamental o conductual y advierten los efectos negativos que podría generar el hecho de que se pretenda limitar su efectividad, pues ello podría generar que aparezca en su lugar más restricciones a través de la regulación estatal que termine afectando o limitando beneficios a los consumidores (Chen y Stallaert, 2010).

\section{CONCLUSIONES}

En este artículo hemos demostrado la importancia de la publicidad programática digital para la competencia y para los consumidores. Dichas estrategias publicitarias, que cada vez son más usadas por los operadores económicos, permiten que los mercados sean más competitivos al permitir posicionar con mayor posibilidad y en menos tiempo a nuevos operadores económicos; y a la vez, trasladan más información a los consumidores, quienes podrán recibir la publicidad que calza de acuerdo con los intereses manifestados a las plataformas que le dirigen publicidad y/o a la data que dicha plataforma posee por terceros. Esto evidentemente genera que los anunciantes tengan mayores posibilidades de éxito en la venta de sus productos o servicios y a un menor costo de inversión, como también permite que los consumidores ahorren costos de transacción en el tiempo de búsqueda que usualmente se tomarían en poder obtener más información sobre los productos que le interesan en el mercado.

Sin perjuicio de ello, como toda estrategia publicitaria que tiene efectos en el mercado, deben evaluarse también los efectos negativos que podría generar si se utilizan de forma desleal o en perjuicio de los consumidores, al infringir sus derechos de información, a no ser discriminados, el uso de sus datos personales y su derecho a la 
privacidad. En el presente trabajo hemos podido apreciar de manera general cómo se vienen resolviendo los conflictos vinculados a la publicidad programática contextual o comportamental a nivel europeo y norteamericano; sin embargo, estas estrategias no dejan de evolucionar a nivel mundial y cada día son más utilizadas por los operadores económicos, que están en proceso de trasladarse completamente a mercados online. Ello explica la relevancia de su estudio y la necesidad de entender el funcionamiento de estas estrategias por parte de reguladores, con el objeto de reducir el riesgo de intervenciones que terminen generando perjuicios para los mercados y consumidores. De este modo, pretendemos que este trabajo sirva al legislador latinoamericano en el entendimiento de la publicidad programática, sus beneficios para el mercado y consumidores, como también los potenciales efectos negativos que podría generar su mal uso.

\section{REFERENCIAS}

Amunátegui Perelló, C. (2006). Los metatags en el comercio electrónico. Un análisis de la jurisprudencia norteamericana y la doctrina española. Revista Cbilena de Derecho, 33.

Carrillo-Durán, M. y Rodrícuez-Silgado, A. (2018). El ecosistema programático. La nueva publicidad digital que conecta datos con personas. El Profesional de la Información, 27(1), 195-201. htps://doi.org/10.3145/epi.2018.ene.18

CHEn, J. y StallaerT, J. (2010). An Economic Analysis of Online Advertising Using Behavioral Targeting. MIS Quarterly, 38(2), 429-449, 2014. https://ssrn.com/abstract=1787608 or http://dx.doi.org/10.2139/ssrn. 1787608

ClickZ. (2019). How Netflix uses big data to create content and enhance user experience. https://www.clickz.com/how-netflix-uses-big-data-content/228201/

FletChER, W. (2010). Advertising A Very Short Introduction. Oxford University Press.

FuEROGHNE, D. (2007). Law \& Advertising - Current Legal Issues for Agencies, Advertisers and Attorneys. Yellow Cat Press.

Garrido Pintado, P., Caerols Mateo, R. y García Huertas, J. G. (2018). Estudio Delphi sobre la evolución y perspectivas de la compra programática de publicidad en España. Doxa Comunicación, 27.

Gracia, J. L., Cebollero, E. y Torelli, V. (2016). Infracción de marca en internet. En LópezTarruella Martínez, Aurelio. Derecho tic Derecho de las tecnologías de la información y de la comunicación. Tirant lo Blanch.

GiL GonZÁles, E. (2016). Big data, privacidad y protección de datos. Agencia de Protección de Datos. JoRdAN, P. (2014). International Advertising Law. A practical Global Guide. Globe Business Publishing Ltd.

KLein, D. y GlaZeR, D. (2003). Reconsidering initial interest confusion on the internet. The Trademark Reporter, 3.

Lastowka, G. (2007). Google's Law. Duke Science, Technology \& Innovation Paper n. 26. https://ssrn.com/abstract=1017536 or http://dx.doi.org/10.2139/ssrn.1017536 
Lerner, A. (2014, 26 de agosto). The Role of 'Big Data' in Online Platform Competition. https://ssrn.com/abstract=2482780 or http://dx.doi.org/10.2139/ssrn. 2482780

LÓPEZ JimÉNEZ, D. (2013). La publicidad en Internet. Regulación y Autorregulación. Aranzadi, S. A. MarisCal, E. y Evans, D. (2012). The Role of Keyword Advertising in Competition among Rival Brands. Coase-Sandor Institute for Law \& Economics Working Paper, n. 619.

Martínez Otero, J. M. y Miralles Pechuán, L. (2014). Revista Aranzadi de Derecho y Nuevas Tecnologías 34, pp. 67-90.

Otamendi, J. (2012). Ley de marcas. Metatags y keywords. En La Ley AR/DOC/1117/2012.

PICKER. (2009). Online Advertising, Identity and Privacy. Law and Economics Working Paper, n. ${ }^{\circ} 475$. PÉREZ BeS, F. (2012). La publicidad comportamental online. El Ciervo 96, S. A.

SÁnChEZ BraVo, G. (2019). La protección de los datos en tiempos del big data y los algoritmos. En Derecho y nuevas tecnologías. El impacto de una nueva era. Themis.

SosA, A. (2020). Uso de marcas de terceros competidores en Google Ads sin autorización en el Perú cinfracción marcaria o acto de competencia desleal? Derecho \& Sociedad, n. 54.

SosA, A. (2018) Publicidad Comparativa en el Perú: una especie en peligro de extinción. Revista Foro Jurídico, año XV, n. ${ }^{\circ} 17$.

TÉlLEZ, E. (2020). Análisis documental sobre el tema del big data y su impacto en los Derechos Humanos. Derecho PUCP, n. ${ }^{\circ}$ 58, p. 165. Pontificia Universidad Católica del Perú.

TOURIÑO, A. (2014). El derecho al olvido y a la intimidad en Internet. Los Libros de la Catarata.

Townley, C., Morrison, E. y Yeung, K. (2017, 6 de octubre). Big Data and Personalised Price Discrimination in EU Competition Law. King's College London Law School Researcb Paper, n. ${ }^{\circ}$

2017-38. https://ssrn.com/abstract=3048688 or http://dx.doi.org/10.2139/ssrn. 3048688

Tucker, C. E. (2016). Social Advertising: How Advertising that Explicitly Promotes Social Influence Can Backfire. https://ssrn.com/abstract=1975897 or http://dx.doi.org/10.2139/ ssrn. 1975897

Uberoi. (2017, 5 de abril). Digital Innovation and transformation. En ZARA: Achieving the "Fast" in Fast Fashion through Analytics. https://digital.hbs.edu/platform-digit/submission/zara-achieving-the-fast-in-fast-fashion-through-analytics/

ZACHARY, J. Z. (2006). Searching for Confusion: The Initial Interest Confusion Doctrine and Its Misapplication to Search Engine Sponsored Links, 91 Cornell L. Rev. 1343.

\section{Documentos}

Comisión Europea (2020, 2 de junio). Unión Europea. En Digital Services Act package: Ex ante regulatory instrument for large online. https:/ec.europa.eu/info/law/better-regulation/ have-your-say/initiatives/12418-Digital-Services-Act-package-ex-ante-regulatoryinstrument-of-very-large-online-platforms-acting-as-gatekeepers

INDECOPI. (noviembre de 2019). Guía de publicidad para influencers. En INDECOPI. https:// www.indecopi.gob.pe/documents/1902049/3749438/Gu\%C3\%ADa+de+Publicidad+pa $\mathrm{ra}+$ Influencers+VF+13.11.19.pdf/66da0113-9071-36a8-da91-a81d123c6a42 


\section{Enlaces web}

Cyberclick Marketing Digital. (2019). Youtube. https://www.youtube.com/ watch? $\mathrm{v}=\mathrm{vVWy}$ GjTsdxw

Facebook (2020, 1 de julio). Información sobre los anuncios de Facebook. https://www.facebook.com/ads/about

\section{Legislación y sentencias}

Asunto C673/17, del 1 de octubre de 2019.

Asunto C324/09, del 12 de julio de 2011.

Asunto C323/09, del 22 de setiembre del 2011.

Reglamento 2016/679 del Parlamento Europeo y del Consejo de 27 de abril de 2016 relativo a la protección de las personas físicas en lo que respecta al tratamiento de datos personales y a la libre circulación de estos datos y por el que se deroga la Directiva 95/46/CE (Reglamento General de Protección de Datos). 